\title{
Significance of Nucleic Acid Testing in Window Period Donations: Revisiting Transfusion Safety in High prevalence-low resource settings
}

\author{
Mangwana $\mathrm{S}^{1}$, Bedi $\mathrm{N}^{1}$ \\ ${ }^{I}$ Department of Blood Transfusion Services, Sri Balaji Action Medical Institute, Paschim Vihar, New Delhi, India
}

\author{
Keywords: \\ HIV; \\ Hepatitis B Surface An- \\ tigen; \\ Hepatitis C Virus, \\ ELISA; \\ Nucleic Acid Amplifica- \\ tion Testing; \\ NAT
}

\begin{abstract}
BACKGROUND: Safe blood transfusion to the people is essential requirement of health care delivery system. Despite the mandatory screening of blood with newest, very sensitive serological test, considerable risk remains for transfusion transmission of virus due to window period infections.

Study was aimed to analyze the efficacy of Mini pool Nucleic Acid Amplification Testing as additional donor screening program and its role in improving blood safety in the high prevalence population.

MATERIAL AND METHODS: Study was performed at a tertiary-care, accreditated hospital from June 2013 to December 2015 All negative cases for anti-HIV, anti-HCV and HBsAg by ELISA were subjected to MP-NAT to detect HIV-1, HIV-2 and HCV-RNA with HBV DNA.

RESULT: In 31 months, 20470 donations were received of which whole blood donations were 16997 $(83.03 \%)$ and 3473 (16.97\%) apheresis. Out of 16997 donations, 446 (2.61\%) were seroreactive. Out of 16551 sero-negative donors subjected to MP-NAT testing, $17(0.10 \%)$ were NAT reactive (NAT yield -1 in 974). Out of 17 cases, 12 were HBV (1 in 1379), four HCV (1 in 4138) and one HIV NAT reactive (1 in 16551).

CONCLUSION: NAT has improvised the blood safety by detecting the virus in the pre-seroconversion, window period thereby providing much higher sensitivity as compared to newest generation serological tests. In countries with high incidence of infection with significant number of window period donations, NAT can serve as a valuable tool along with other serological testing in high prevalence, resource constrained countries to achieve the goal of zero risk of blood.
\end{abstract}

\section{INTRODUCTION}

Safe blood transfusion to the people is essential requirement of health care delivery system which can be done

\footnotetext{
Correspondence:

Dr Mangwana Sadhana, MD.

Department of Blood Transfusion Services, Sri

Balaji Action Medical Institute, New Delhi, India

E-mail: sadhanamangwana@yahoo.co.in
}

through integrated strategy for elimination of transfusion transmitted infection. Safe blood can be ensured through collection of blood from voluntary, non-remunerated blood donor and screening of all donations for viral markers by highly sensitive tests. Family / replacement constitute the largest group of blood donors in India. ${ }^{1-3}$ Voluntary nonremunerated blood donations in India have raised to $84 \%$ by September $2014 .{ }^{4}$

According to Joint United Nations Programme on HIV / 
AIDS (UNAIDS) global report 2015, 36.9 million (34.3 million - 41.4 million) people globally are living with Human Immunodeficiency Virus(HIV) while in Asia and pacific 5 million (4.5 million-5.6 million) people are living with HIV. ${ }^{5}$ China, Indonesia and India account for $78 \%$ of new infections in the region. ${ }^{5}$ National adult (1549 yrs) HIV prevalence is $0.26 \%(0.22-0.32 \%)$ in $2015^{6}$ with HIV seroreactivity as $0.2 \%$ in blood donations in NACO supported blood banks. ${ }^{4}$ India is considered to have intermediate level of Hepatitis B Virus (HBV) endemicity ${ }^{7}$ having $3-4.2 \%$ of prevalence with more than 40 million HBV carriers. ${ }^{8}$ Hepatitis C Virus (HCV) infection in India has a population prevalence of $1 \% .{ }^{9}$

With increasing voluntary blood donations, prevention of transmission of infectious disease through blood transfusion is challenge in developing, low resource countries. Supply of risk free blood is a challenge and policies and strategies to reduce the risk of Transfusion Transmitted Infection need to be augmented.

Despite the mandatory screening of blood with newest, very sensitive serological test, considerable risk remains for transfusion transmission of virus due to window period infections, occult infections, viral variants or delayed seroconversion. It is now well established that Nucleic Acid Testing (NAT) reduces the window period of Transfusion Transmitted Infection and helps improve blood safety. ${ }^{10}$ NAT is used in conjunction with serological tests. NAT can be done individually or in pools.

The primary objective of this study is to analyze the efficacy of MP-NAT testing as additional donor screening programme, seroprevalence of transfusion transmitted infections (TTI) and its role in improving blood safety in the high prevalence population coming to a tertiary care, super- specialty hospital.

\section{MATERIALS AND METHODS}

The study was performed at a tertiary-care, NABH accredited hospital from June 2013 to December 2015. At the time of blood collection, donor samples were collected for all mandatory screening serological tests and MP-NAT Testing. ELISA Tests for HIV, HBsAg and HCV were done on Automated EVOLIS System (Bio-Rad). All negative cases were subjected to MP-NAT in small pools of six on Roche's Cobas Taq Screen MPX assay v2.0 on Cobas System s 201(Roche Diagnostics Gmbh, Mannheim) to detect HIV-1 (groups M and O RNA), HIV-2 RNA, HCVRNA and HBV DNA. The Cobas Taq Screen MPX assay comprises of four automated steps as follows-
i. Pooling of samples
ii. Sample preparation
iii. Real time PCR amplification and detection.
iv. Data management and Reporting.

The test incorporates an internal control for monitoring test performance in each individual test as well as Amperase enzyme to reduce potential contamination by previously amplified material. Reactive (created) pools were retested individually to confirm and to know the infection in donor sample. Limits of detection (with $95 \%$ probabit) for various analytes on Taqscreen MPX v 2.0 are as - HIV -1 Group M - 46.2 IU/mL, HIV -1 Group O - 18.3 Copies /ml, HIV-256.2 copies /ml, HCV-6.8 IU /mL, HBV- $2.3 \mathrm{IU} / \mathrm{mL}$. HIV1 Group $\mathrm{M}, \mathrm{HCV}$ and $\mathrm{HBV}$ are calibrated against WHO International Standards while HIV-1 Group O and HIV-2 are calibrated against FDA Reference reagents.

\section{RESULTS}

A total of 20470 donations were received over the period of 31 months between June 2013 to December 2015 of which 19932 (97.37\%) were males and 538 (2.63\%) were females. $16997(83.03 \%)$ were whole blood donations and $3473(16.97 \%)$ were apheresis. 779 (3.80\%) were voluntary donors and $19691(96.20 \%)$ were replacement donors. Out of 16997 whole blood donations, 446 (2.61\%) were seroreactive of which $139(0.68 \%)$ were HBsAg seroreactive donors, 60 (0.29\%) were anti-HCV seroreactive, $36(0.18 \%)$ were anti-HIV seroreactive and $211(1.03 \%)$ were anti-TP (Syphilis) seroreactive.

Sixteen thousand, five hundred and fifty one sero-negative donors' samples were subjected to MP-NAT testing of which $17(0.10 \%)$ were NAT reactive. Out of 17 NAT yield (Seronegative / NAT reactive), 12 (0.07\%) were HBV NAT reactive, four $(0.024 \%) \mathrm{HCV}$ NAT reactive and one $(0.006 \%)$ HIV NAT reactive. No sample showed coinfection. All NAT yield donors were males, replacement donors. Donors' age ranged from 19 years -49 years.

\section{DISCUSSION}

With high prevalence of HIV, HBV and HCV in India, safe blood transfusion is a big challenge for the health care system. Transfusion transmitted infections continue to be the biggest threat to the blood transfusion safety. Despite the advancements made in the serological testing of $\mathrm{HBV}$, $\mathrm{HCV}$ and HIV, significant transfusion transmission of virus still remains.

New technology like NAT has improvised the blood safety by detecting the virus in the pre-sero conversion window period and thereby providing the sensitivity that is much higher compared to the newest generation serological tests. Though the NAT technology is widely used in the developed countries but the use is limited to a few centers only in high prevalence low resource countries like India.

Our study has shown predominance of replacement donors (96.2\%) similar to other studies ${ }^{2,11-13}$ where $98.08 \%, 82.4 \%$, $94.7 \%$ and $99.48 \%$, donors respectively were replacement donors. High number of replacement donors reflects lack of awareness in general population, misconception and fears for blood donations and lack of health education. ${ }^{2}$ Family 
donors are not safe as voluntary donors and voluntary, non-remunerated, repeat blood donors are perceived to be safer than the first time blood donors., ${ }^{3,14}$ In this study, all NAT yield cases were replacement donors. There were no voluntary donors.

The overall seropositivity rate in this study of 31 months was around $2.61 \%$ which is slightly higher than our previous study of seven years seroprevalence which was $2.11 \%$ before inclusion of NAT technology. ${ }^{2}$ This marginal increase in overall seroprevalence could be due to detection of window period infections which were not detectable by routine serological tests. This technology limitation puts transfusion recipients to risk for transmissible diseases. The seroprevalence for HIV, HBV and HCV for this study period was $0.18 \%, 0.68 \%$, and $0.29 \%$ respectively which was lower than the overall prevalence of the same in India i.e. $0.26 \%, 3-4.2 \%$, and $1 \%$ respectively. ${ }^{6,8,9}$

Screening for HIV, HBV and HCV in India is based mainly on serological testing with few centers introducing NAT testing. Most populations in resource limited countries suffer from high prevalence of transfusion transmitted infections and are expected to have more frequent incident and occult cases. ${ }^{15}$ Of the 16551 seronegative donor's samples subjected to NAT testing, there were 17 NAT yields ( 1 in 974). The NAT yield rate from other blood Centers in India is 1 in $753,{ }^{16} 1$ in $610,{ }^{17} 1$ in $1528,{ }^{18} 1$ in $2622,{ }^{19} 1$ in $2000,{ }^{20}$ and 1 in $2972 .{ }^{15}$ The higher NAT yield compared to other studies $15,18,19,20$ is probably on account of higher prevalence in the population reaching to this tertiary care hospital.

Amongst these 17 cases of NAT yield (1 in 974), HBV NAT yield was 1 in 1379 donations which was almost similar to the yield shown by other studies i.e. 1 in $1012^{17}$ and 1 in 1221. ${ }^{16}$ Our HBV NAT yield was higher than those found by Chigurupati et al ( 1 in 2000) $)^{20}$ and Chandrashekhar ( 1 in $26630)^{21}$ which might be due to higher prevalence of HBV in our population.

HBV NAT yield of 1 in 1379 is much higher than studies done in Europe and USA where reported prevalence is $1: 600,000$ to $1: 350,000 .^{22}$ Similarly, higher prevalence of HIV-1 and HCV in India as compared to western Europe and USA $(1: 300,000 \text { to } 1: 3,000,000)^{22,23}$ are leading to increased $\mathrm{HCV}$ and HIV-1 yield cases as also seen in this study (1 in 4138 and 1 in 16551 respectively) and by Aggarwal et al (HCV yield- 1 in 1997) ${ }^{17}$ and Kumar et al ( HCV yield- 1 in 2536). ${ }^{16}$ The plausible explanation for this variation in the yield rate could be due to high prevalence of HBV and $\mathrm{HCV}$ in the population as India is considered to be in the intermediate level of HBV endemicity with over 40 million HBV carriers $^{8}$ and lesser number of voluntary donations. This has shown a clear advantage of cost effectiveness of NAT screening in developing over developed countries. Developed countries are spending resources on NAT screening to detect only one window period donation in 300,000 to $3,000,000$ donations saving three lives while in developing countries with high NAT yield of 1 in 974 (as in this study), NAT screening is 300 to 3000 times more beneficial as compared to developed countries saving much more lives which, in turn, is more cost effective.

This study has certain limitations as viral load through quantitative polymerase chain reaction (PCR) couldn't be performed. The donors in window period donations could not be followed for seroconversion.

However, NAT test with pooling strategies integrated with antibody testing has revolutionized the blood safety by intercepting potentially infectious pathogens. Since viremia precedes seroconversion by several days to weeks, Nucleic Acid Amplification test (NAT) technologies have the potential to detect viremia earlier than routine screening tests based on seroconversion. In countries with high incidence of infection with significant number of window period donations, NAT can serve as a valuable tool to achieve the goal of zero risk of blood. It should be kept in mind that incremental cost- effectiveness of NAT is marginal as compared to safety benefits along with direct cost of medications, monitoring, medical care of the infected person and financial loss of monthly earning as well as other family members of the affected person.

\section{CONCLUSION}

With high prevalence of all TTIs in India, implementation of NAT would save more lives of patients and of window period donors by their counselling, early referral to the physicians for close follow up for seroconversion and treatment; further adding the advantage. With clearer benefits of NAT outweighing cost in providing medical care to the infected person, it is recommended that NAT should be made mandatory by policymakers as a part of TTI testing along with other serological testing to provide safe blood in high prevalence, resource constrained countries like India and it can be a step towards zero risk of blood.

\section{REFERENCES}

1. Makroo RN, Salil P, Vashist RP, Shivlal. Trends of HIV infection in the blood donors of Delhi. Indian J Pathol Microbiol. 1996;39:13942. Crossref

2. Mangwana S, Arya D, Yadav P. Safety of Blood and Blood Components: Analysis of Seroprevalence and Trends of HIV, HBV and HCV. International Journal of Basic and Applied Medical Sciences. Available from : Crossref

3. Jain R, Gupta G. Family/friend donors are not true voluntary donors. Asian J Transfus Sci. 2012;6:29-31. Crossref

4. Naco Annual Reportn2014-2015. Available from: Crossref

5. UNAIDS factsheet 2015. Available from: Crossref

6. India HIV estimation 2015. Technical report. Available from: Crossref 
7. NCDC letter (2014). Available from : Crossref

8. WHO $\mid$ Hepatitis B Media Centre Fact Sheet N0 204 (2015). Available from: Crossref . [Cited on 6th January 2016]

9. WHO Hepatitis C Media Centre Fact Sheet N0 164 (2015). Available from: Crossref . [Cited on 6th January 2016]

10. Pondé RA. Genomic detection of human immunodeficiency virus (HIV) by nucleic acid amplification test in a frequent platelet donor during the pre-seroconversion period. Arch Virol. 2011;156:2085-90. Crossref

11. Singh B, Verma M, Kotru M, Verma K, Batra M. Prevalence of HIV and VDRL seropositivity in blood donors of Delhi. Indian J Med Res. 2005; 122:234-6. Crossref

12. Kakkar N, Kaur R, Dhanoa J. Voluntary donors-need for a second look. Indian J Pathol Microbiol. 2004;47:381-3. Crossref

13. Pahuja S, Sharma M, Baitha B, Jain M. Prevalence and trends of markers of hepatitis $\mathrm{C}$ virus, hepatitis $\mathrm{B}$ virus and human immunodeficiency virus in Delhi blood donors: a hospital based study. Jpn J Infect Dis. 2007;60:389-91. Crossref

14. Choudhury N, Tulsiani S, Desai P, Shah R, Mathur A, Harimoorthy $\mathrm{V}$. Serial follow-up of repeat voluntary blood donors reactive for antiHCV ELISA. Asian J Transfus Sci. 2011;5:26-31. Crossref

15. Jain R, Aggarwal P, Gupta GN. Need for nucleic Acid testing in countries with high prevalence of transfusion-transmitted infections. ISRN Hematol.2012; 2012:718671. Crossref

16. Kumar R, Gupta S, Kaur A, Gupta M. Individual donor-nucleic acid testing for human immunodeficiency virus-1, hepatitis $\mathrm{C}$ virus and hepatitis B virus and its role in blood safety. Asian J Transfus Sci. 2015;9:199-202. Crossref

17. Agarwal N, Chatterjee K, Coshic P, Borgohain M. Nucleic acid testing for blood banks: an experience from a tertiary care centre in New Delhi, India. Transfus Apher Sci. 2013;49:482-4. Crossref

18. Makroo RN, Choudhury N, Jagannathan L et al. Multicenter evaluation of individual donor nucleic acid testing (NAT) for simultaneous detection of human immunodeficiency virus -1 and hepatitis B and C viruses in Indian blood donors. Indian J Med Res. 2008;127:140-7. Crossref

19. Chatterjee $\mathrm{K}$, Coshic $\mathrm{P}$, Borgohain $\mathrm{M}$ et al. Individual donor nucleic acid testing for blood safety against HIV-1 and hepatitis B and C viruses in a tertiary care hospital. Natl Med J India. 2012;25:207-9. Crossref

20. Chigurupati P, Murthy KS. Automated nucleic acid amplification testing in blood banks: An additional layer of blood safety. Asian J Transfus Sci. 2015;9:9-11. Crossref

21. Chandrashekar S. Half a decade of mini-pool nucleic acid testing: Cost-effective way for improving blood safety in India. Asian J Transfus Sci.2014;8:35-8. Crossref

22. B. Singh. "Nucleic Acid Testing (NAT) screening of blood donors in India" A Project Report, International Hospital Federation Reference Book, 2008/2009.

23. Kleinman SH, Strong DM, Tegtmeier GG et al. Hepatitis B virus (HBV) DNA screening of blood donations in minipools with the COBAS AmpliScreen HBV test. Transfusion. 2005;45:1247-57. Crossref 